\title{
Diagonal Multi-Antenna Coded Caching for Reduced Subpacketization
}

\author{
MohammadJavad Salehi and Antti Tölli \\ Center for Wireless Communications, University of Oulu, 90570 Finland \\ Email: \{fist_name.last_name\}@oulu.fi
}

\begin{abstract}
Large subpacketization requirement is known to be a significant issue in the practical implementation of multiantenna coded caching schemes. In this paper, we introduce a new class of schemes applicable to any network in which the spatial degrees of freedom $\alpha$ is larger than or equal to the coded caching gain. This class of schemes, called diagonal coded caching (DCC), is based on a tabular representation of transmitted codewords and includes consecutive diagonal shifting of such representations over a well-defined placement table. We find the lower-bound for the required subpacketization for any DCC scheme and show that it is achievable using simple, novel algorithms. This not only enables smaller subpacketization compared to state of the art but also makes it possible to control the subpacketization by carefully adjusting the $\alpha$ parameter.
\end{abstract}

Index Terms - coded caching, multi-antenna communications, subpacketization

\section{INTRODUCTION}

Network data volume has continuously grown during the past years. The global IP (Internet Protocol) data volume is expected to exceed 4.8 Zettabytes $\left(10^{21}\right.$ bytes) by 2022 , from which 71 percent will pass through wireless networks [1]. Mobile video applications account for a significant part of this data volume, as their share is expected to reach $79 \%$ of the global mobile data traffic by 2022. Consequently, great efforts are made by the research community for developing new communication schemes well-suited to current and future (e.g., immersive viewing) video applications.

Most video applications share a few crucial features. First, the received content source is not important, as long as each user receives its requested content [2]. Moreover, the content request probability distribution is uneven, and there is a prime time where the request rate is high [3]. As a result, caching popular content is considered a viable solution for large scale video delivery [2], [4], especially considering the continuous decline in the price of memory chips [5].

Recently, Coded Caching (CC) is introduced as a promising extension to conventional caching schemes [6]. It enables a global caching gain, proportional to the total cache size in the network, to be achieved in addition to the local caching gain at each user. This extra gain is enabled by multicasting carefully designed codewords to various user subsets, such that each codeword contains useful data for all users in its target subset. It is also shown that $\mathrm{CC}$ gain is additive with the spatial gain of using multiple antennas [7], [8], making $\mathrm{CC}$ even

This work was supported by the Academy of Finland under grants no. 319059 (Coded Collaborative Caching for Wireless Energy Efficiency) and 318927 (6Genesis Flagship). more desirable for future networks in which multi-input multioutput (MIMO) communications play an important role [9].

Despite its benefits, CC still requires essential issues to be solved before it can be practically implemented. In this paper, we target one such issue, known as the large subpacketization requirement. The problem is that the number of smaller parts each file is split into, known as subpacketization, grows exponentially with the user count $K$, making $\mathrm{CC}$ implementation infeasible, even for networks with a moderate size [10]. In this paper, we introduce a new class of CC schemes for multiantenna communication setups. Schemes within this class are applicable to networks in which the spatial degrees of freedom $(\mathrm{DoF}) \alpha$ is larger than or equal to the $\mathrm{CC}$ gain $t$. We first show that the state of the art low-subpacketization scheme introduced in [11] belongs to this class. Then, we provide a lower-bound on the required subpacketization for any scheme within this class and show that this lower-bound is not only achievable but can also be controlled through careful adjustment of the spatial DoF. The result is a class of efficient schemes with adjustable, smaller than the state of the art subpacketization requirements. This is a concrete solution to CC schemes' subpacketization bottleneck, making CC one step closer to practical implementation in future networks.

In this paper, we use $[K]$ and $[i: j]$ to denote $\{1,2, \ldots, K\}$ and $\{i, i+1, \ldots, j\}$, respectively. Vectors are represented by boldface lower-case letters. The element at index $i$ of a vector $\mathbf{v}$ is shown by $\mathbf{v}[i]$. Sets are denoted by calligraphic letters, and for a set $\mathcal{A},|\mathcal{A}|$ represents the number of elements in $\mathcal{A}$. Upper-case sans-serif letters are used to indicate tables. A point is shown by $\mathrm{a}=(p, k)$ and refers to the cell in the $p$-th row and $k$-th column of any given table. The value of point $a$ in table $\mathrm{T}$ is shown by $\mathrm{T}[\mathrm{a}]$.

\section{SySTEM MODEL}

We consider a multiple-input single-output (MISO) setup, where a single server communicates with $K$ users over a shared wireless link with the capacity of one data unit per channel use. The server has $L$ transmitting antennas, and each user is equipped with a single antenna. In order to make the setup more general, we assume the spatial DoF can be freely adjusted as $\alpha \leq L$. In [12] it is shown that smaller $\alpha$ not only simplifies the beamformer design but also enables a better beamforming gain, resulting in an improved performance at the finite-SNR regime. We also assume full channel state information (CSI) is available at the server, and it has access to a library of $N \geq K$ files, denoted by $\mathcal{F}$. Each file $W \in \mathcal{F}$ 
has a size of one data unit and each user is equipped with a cache memory of size $M$ data units.

The system operation consists of two distinct phases, placement, and delivery. During the placement phase, which occurs at the low network traffic time, the users' cache memories are filled by data from the files in $\mathcal{F}$. This is done according to a cache placement algorithm, which operates without any prior knowledge of file request probabilities in the delivery phase. We use $\mathcal{Z}(k)$ to denote the cache contents of user $k$ after the placement phase is completed.

At the beginning of the delivery phase, each user $k$ reveals its requested file $W(k) \in \mathcal{F}$. Let us define the demand set as $\mathcal{D}=\{W(k) \mid k \in[K]\}$. Based on $\mathcal{D}$ and following a delivery algorithm, the server builds a set of transmission vectors, each with dimensions $L \times 1$, and transmits them in consecutive time intervals. Transmission vectors are built such that each user $k$ can decode $W(k)$, using $\mathcal{Z}(k)$ (locally cached data) together with the data received from the channel.

We assume zero-forcing (ZF) beamformers are used to remove (part of) the inter-stream interference ${ }^{1}$. A $\mathrm{ZF}$ vector is denoted by $\mathbf{w}_{\mathcal{R}} \in \mathbb{C}^{L}$, where $\mathcal{R} \subseteq[K]$ is the ZF set with $|\mathcal{R}|=\alpha-1$. Defining $\mathbf{h}_{k} \in \mathbb{C}^{L}$ as the channel vector from $L$ transmitting antennas to the user $k, \mathbf{w}_{\mathcal{R}}$ is built such that $\mathbf{h}_{k}^{H} \mathbf{w}_{\mathcal{R}}=0$, for every $k \in \mathcal{R}$. For simplicity, we ignore the brackets while mentioning the set $\mathcal{R}$; e.g. $\mathbf{w}_{1,2} \equiv \mathbf{w}_{\{1,2\}}$.

Tabular Summation. Any cell in a table may contain an integer number or a special value $c$. Considering two tables $\mathrm{A}$ and $\mathrm{B}$, both with dimensions $P \times K, \mathrm{Y}=\mathrm{A}+\mathrm{B}$ is a new $P \times K$ table resulting from element-wise summation of $\mathrm{A}$ and B. We treat the special value $c$ as a general number; e.g. as a result of summation between $c$ and 1 we simply put $c+1$, and for $c+c$ we simply use $2 c$.

\section{STATE OF THE ART}

\section{A. Coded Caching}

The original $\mathrm{CC}$ scheme provided in [6] achieves the total DoF of $t+1$ in a single-antenna setup, where $t=\frac{K M}{N}$ is the $\mathrm{CC}$ gain. The subpacketization requirement for this scheme is $\left(\begin{array}{c}K \\ t\end{array}\right)$. This scheme is later extended in various directions. In one interesting extension, in [7] it is shown that the $\mathrm{CC}$ gain is additive with the multi-server gain; i.e. the total DoF of $t+L$ is achievable with $L$ transmitting servers. However, the multi-server scheme in [7] needs a larger subpacketization of

$$
\left(\begin{array}{c}
K \\
t
\end{array}\right)\left(\begin{array}{c}
K-t-1 \\
L-1
\end{array}\right) \text {. }
$$

Following [7], applying CC to multi-antenna setups is studied in [8]. The scheme of [8] is a direct extension of [7], where a transmitting antenna replaces each server and the interference is nulled out using ZF beamformers. As a result, it has the same subpacketization requirement of (1).

$\mathrm{ZF}$ beamformers are not an appropriate choice for finiteSNR communications. In [12], [13] it is shown that controlling

\footnotetext{
${ }^{1}$ Note that all the methods and results in this paper are still valid for other beamformer types (e.g., MMSE-type) and noisy channel estimates. However, as the paper aims to reduce subpacketization without the DoF loss, the proposed simplistic model is considered here.
}

interference by using fully optimized beamformers results in a much better rate performance at finite-SNR. However, such beamformers require non-convex optimization problems to be solved. In [12], successive convex approximation (SCA) is used to solve this issue, and the complexity is controlled by two parameters $\alpha$ and $\beta$. In fact, $\alpha$ controls the spatial DoF (same as the current paper), while $\beta$ controls the overlap of parallel multicast messages at each user. However, the subpacketization requirement is further increased to

$$
\frac{(\alpha-1) !}{(\delta-1) !(\beta-1) !(t+\beta) ! \delta-1}\left(\begin{array}{c}
K \\
t
\end{array}\right)\left(\begin{array}{c}
K-t-1 \\
L-1
\end{array}\right),
$$

where $\delta=\frac{t+\alpha}{t+\beta}$. Unfortunately, the large subpacketization requirement seriously limits the applicability of all these schemes [10]. Consequently, reducing subpacketization has been extensively studied in the literature, for both single- and multi-antenna cache-enabled setups.

\section{B. Subpacketization in Single-Antenna Coded Caching}

Subpacketization is well-studied for single-antenna cacheenabled communications. For centralized schemes, in [14], Placement Delivery Array (PDA) is presented as a systematic approach to reducing subpacketization. It is shown that the original scheme of [6] is a PDA-driven scheme and is optimal among one symmetric class of schemes known as $g$-regular PDA. In [15], it is shown that, if the rate is constant, a PDA resulting in linear subpacketization does not. In [16] a sub-exponential subpacketization scheme achieving fixed rate for constant $M / N$ value is proposed. In [17], RuzsaSzeméredi graphs are used to design CC schemes with linear subpacketization growth, but with non-constant rate.

\section{Subpacketization in Multi-Antenna Coded Caching}

For multi-antenna setups, in [10] it is shown that if $K / L$ and $t / L$ are both integers, any single-antenna scheme with subpacketization $g(K, t)$ has a multi-antenna counterpart, with subpacketization $g(k / L, t / L)$ and without any DoF loss ( $g$ is a general function). For example, the scheme of [6] can be applied to multi-antenna setups with the subpacketization requirement of $\left(\begin{array}{c}K / L \\ t / L\end{array}\right)$. However, the proposed elevation scheme suffers DoF loss (and increased subpacketization) if either $K / L$ or $t / L$ is non-integer. Specifically, DoF is reduced by a multiplicative factor (gap), bounded above by 2 when $L>t$, and by $\frac{3}{2}$ when $L<t$.

In [18], a CC scheme with flexible subpacketization is introduced, and it is shown that using optimized beamformers, larger subpacketization enables a better rate performance. The results are limited to the specific case of $K=t+L$, however. In [11] a new scheme with subpacketization requirement of $K(t+L)$ is introduced. This scheme, applicable to any cacheenabled setup with $L \geq t$, enables linear subpacketization (or quadratic, based on how $t$ scales with $K$ ) for a large set of cache-enabled setups. However, it lacks a discussion on the possible optimality of the proposed scheme.

\section{Our Contribution}

We introduce a new class of CC schemes applicable to networks in which the total available DoF $t+\alpha$ is smaller than 
the user count $K$. For the case $K=t+\alpha$, the subpacketization issue is well-studied in [18]. Also, if $K<t+\alpha$, one can still use the scheme of [18] by using the excess DoF to enhance the beamforming gain. We also assume that the spatial DoF $\alpha$ is larger than or equal to the $\mathrm{CC}$ gain $t$. For the case $t>\alpha$, by accepting moderate DoF loss, one can use the $\mathrm{CC}$ scheme in [10] to reduce the subpacketization requirement.

The new class of $\mathrm{CC}$ schemes, referred to as diagonal coded caching (DCC), provides a systematic approach for reducing subpacketization in cache-enabled multi-antenna communication setups. We show that the scheme of [11] is, in fact, a particular member of DCC. We also find the minimum possible subpacketization for any scheme belonging to DCC and show that it is achievable using well-defined algorithms. Last but not least, we discuss how $\alpha$ can be effectively tuned in order to control the subpacketization requirement.

\section{Diagonal Coded CACHING}

\section{A. Placement Table}

In order to describe the DCC structure, we use a placement table T, with $P$ rows and $K$ columns. $P$ can be any integer such that both $\lambda=\frac{K}{P}$ and $\frac{t}{\lambda}$ are integers (clearly, if $P=K$, $\lambda=1$ and both conditions are met). The first row of $\mathrm{T}$ has $t$ cells with $c$ value, and the other cells are filled up with zeros. Each row is a circular shift of the previous row by $\lambda$ steps. Moreover, all the rows of $\mathrm{T}$ are unique.

Example 1. Assume a simple network with $K=4, t=1$ and $\alpha=2$. A placement table for this network can be built as ${ }^{2}$

$$
\mathrm{T}=\begin{array}{|c|c|c|c|}
\hline c & 0 & 0 & 0 \\
\hline 0 & c & 0 & 0 \\
\hline 0 & 0 & c & 0 \\
\hline 0 & 0 & 0 & c \\
\hline
\end{array} .
$$

As presented earlier, the value of point $\mathrm{a}$ in table $\mathrm{T}$ is shown by $\mathrm{T}[\mathrm{a}]$. For example, for table $\mathrm{T}$ in $(3), \mathrm{T}[(1,1)]=c$.

\section{B. Cache Placement}

Given a placement table T with $P$ rows, each file $W$ is first split into $P$ equal-sized packets $W_{p}$. Each $W_{p}$ is then split into $Q$ equal-sized subpackets $W_{p}^{q}$, where the possible values for $Q$ will later be discussed. Subpacketization is defined as $S=P \times Q$, and the goal is to reduce $S$. During the placement phase, for every $p \in[P]$ and $k \in[K]$, if $\mathrm{T}[(p, k)]=c$ we store $W_{p}^{q}$ in the cache memory of user $k$, for every $W \in \mathcal{F}$ and $q \in[Q]$. For example, considering the placement table $\mathrm{T}$ in (3), if $\mathcal{F}=\{A, B, C, D\}$, the cache content of user 1 is

$$
\mathcal{Z}(1)=\left\{A_{1}^{q}, B_{1}^{q}, C_{1}^{q}, D_{1}^{q} \mid q \in[Q]\right\} .
$$

Note that the provided placement scheme meets the cache memory size constraints. This is because in each column (i.e. for each user) there exist $\frac{P t}{K}$ cells with value $c$, where each $c$ is equivalent to caching data parts of size $\frac{1}{P}$ for $N$ files. So the total required cache size, denoted by $\hat{M}$ becomes

$$
\hat{M}=\frac{P t}{K} \times N \times \frac{1}{P}=\frac{N t}{K}=M .
$$

\footnotetext{
2 The procedure outlined in Examples 1-6 requires larger subpacketization compared to the multi-server scheme in [7]. It is used to clarify the concept.
}

\section{Delivery Sets}

In DCC, delivery procedure is based on delivery sets. Given the placement table $\mathrm{T}$, a delivery set $\mathcal{A}$ is a set of $t+\alpha$ points $\mathrm{a}_{i}=\left(p_{i}, k_{i}\right)$ in $\mathrm{T}$. The cover set of $\mathcal{A}$ is defined as

$$
\operatorname{cvr}(\mathcal{A})=\bigcup_{\mathrm{a}_{i} \in \mathcal{A}}\left\{k_{i}\right\} \text {. }
$$

The delivery set $\mathcal{A}$ is called primary, if the following primal conditions hold:

$$
\begin{aligned}
& \text { 1) }|\operatorname{cvr}(\mathcal{A})|=t+\alpha ; \\
& \text { 2) for every } \mathrm{a}_{i} \in \mathcal{A}, \mathrm{T}\left[\mathrm{a}_{i}\right]=0 ; \\
& \text { 3) for every } \mathrm{a}_{i} \in \mathcal{A}, \sum_{k \in \operatorname{cvr}(\mathcal{A})} \mathrm{T}\left[\left(p_{i}, k\right)\right]=t c .
\end{aligned}
$$

A primary delivery set $\mathcal{A}$ is equivalent to a unique transmission vector $\mathbf{x}(\mathcal{A})$, using which every user in $\operatorname{cvr}(\mathcal{A})$ receives part of its requested data interference-free. In fact, for every point $\mathrm{a}_{i}=\left(p_{i}, k_{i}\right)$ in $\mathcal{A}$, a portion of $W_{p_{i}}\left(k_{i}\right)$ is included in $\mathrm{x}(\mathcal{A})$. According to the third primal condition, this data portion is available in the cache memories of $t$ users in $\operatorname{cvr}(\mathcal{A})$, and should be zero-forced at $\alpha-1$ other users, for all the interference to be eliminated. Indeed, as the spatial DoF is assumed to be $\alpha$, zero-forcing at $\alpha-1$ users is possible.

Example 2. Consider the network and the placement table in Example 1. Consider the three points $\mathrm{a}_{1}=(2,1), \mathrm{a}_{2}=(1,2)$ and $\mathrm{a}_{3}=(1,3)$ in $\mathrm{T}$. As $t=1$ and $\alpha=2, \mathcal{A}=\left\{\mathrm{a}_{1}, \mathrm{a}_{2}, \mathrm{a}_{3}\right\}$ is a delivery set. Its cover set is

$$
\operatorname{cvr}(\mathcal{A})=\bigcup_{\mathrm{a}_{i} \in \mathcal{A}}\left\{k_{i}\right\}=\{1,2,3\} .
$$

On the other hand, $\mathrm{T}\left[\mathrm{a}_{i}\right]=0$ for $i \in[3]$ and

$$
\begin{aligned}
& \mathrm{T}[(2,1)]+\mathrm{T}[(2,2)]+\mathrm{T}[(2,3)]=c, \\
& \mathrm{~T}[(1,1)]+\mathrm{T}[(1,2)]+\mathrm{T}[(1,3)]=c,
\end{aligned}
$$

which means $\mathcal{A}$ is a primary delivery set. The transmission vector $\mathrm{x}(\mathcal{A})$ is built as

$$
\mathbf{x}(\mathcal{A})=\mathbf{w}_{3} \hat{W}_{2}(1)+\mathbf{w}_{3} \hat{W}_{1}(2)+\mathbf{w}_{2} \hat{W}_{1}(3),
$$

where $\hat{W}_{p}(k)$ denotes part of the packet $W_{p}(k)$. We will discuss this later when we introduce the subpacket index $q$.

We define a tabular representation $\mathrm{T}_{\mathcal{A}}$, for a primary delivery set $\mathcal{A}$. It is a $P \times K$ table in which for every $\mathrm{a}_{i} \in \mathcal{A}$ we have $\mathrm{T}_{\mathcal{A}}\left[\mathrm{a}_{i}\right]=1$, and all other cells are filled with zero.

Example 3. Consider the network in Example 1. For this network, for the primary delivery set $\mathcal{A}$ in (8), $\mathrm{T}_{\mathcal{A}}$ is

$$
\mathrm{T}_{\mathcal{A}}=\begin{array}{|l|l|l|l|}
\hline 0 & 1 & 1 & 0 \\
\hline 1 & 0 & 0 & 0 \\
\hline 0 & 0 & 0 & 0 \\
\hline 0 & 0 & 0 & 0 \\
\hline
\end{array}
$$

A secondary delivery set $\overline{\mathcal{B}}$ consists of $J$ primary delivery sets $\mathcal{A}_{j}$, where $J$ is called the order of $\overline{\mathcal{B}}$ and its set of possible values is discussed later. Tabular representation of $\overline{\mathcal{B}}$ is denoted by $\mathrm{T}_{\overline{\mathcal{B}}}$ and is calculated as

$$
\mathrm{T}_{\overline{\mathcal{B}}}=\sum_{\mathcal{A}_{j} \in \overline{\mathcal{B}}} \mathrm{T}_{\mathcal{A}_{j}} .
$$

Clearly, a secondary delivery set of order $J$ is equivalent to $J$ transmission vectors $\mathbf{x}\left(\mathcal{A}_{j}\right)$, where $\mathcal{A}_{j} \in \bar{B}$. 
Example 4. Consider the network in Example 1, and the secondary delivery set $\overline{\mathcal{B}}=\left\{\mathcal{A}_{1}, \mathcal{A}_{2}, \mathcal{A}_{3}\right\}$, where

$$
\begin{aligned}
& \mathcal{A}_{1}=\{(2,1),(1,2),(1,3)\}, \\
& \mathcal{A}_{2}=\{(3,1),(1,3),(1,4)\}, \\
& \mathcal{A}_{3}=\{(4,1),(1,4),(1,2)\} .
\end{aligned}
$$

Then $\overline{\mathcal{B}}$ is equivalent to the transmission vectors

$$
\begin{aligned}
& \mathbf{x}\left(\mathcal{A}_{1}\right)=\mathbf{w}_{3} \hat{W}_{2}(1)+\mathbf{w}_{3} \hat{W}_{1}(2)+\mathbf{w}_{2} \hat{W}_{1}(3), \\
& \mathbf{x}\left(\mathcal{A}_{2}\right)=\mathbf{w}_{4} \hat{W}_{3}(1)+\mathbf{w}_{4} \hat{W}_{1}(3)+\mathbf{w}_{3} \hat{W}_{1}(4), \\
& \mathbf{x}\left(\mathcal{A}_{3}\right)=\mathbf{w}_{2} \hat{W}_{4}(1)+\mathbf{w}_{2} \hat{W}_{1}(4)+\mathbf{w}_{4} \hat{W}_{1}(2),
\end{aligned}
$$

and the tabular view of $\overline{\mathcal{B}}$ is

$$
\mathrm{T}_{\overline{\mathcal{B}}}=\begin{array}{|l|l|l|l|}
\hline 0 & 2 & 2 & 2 \\
\hline 1 & 0 & 0 & 0 \\
\hline 1 & 0 & 0 & 0 \\
\hline 1 & 0 & 0 & 0 \\
\hline
\end{array} .
$$

\section{Delivery Phase}

The delivery phase of DCC consists of $P$ transmission rounds, where the transmission round $y$ is based on the secondary delivery set $\overline{\mathcal{B}}^{y}(y \in[P])$. We denote the primary delivery sets in $\overline{\mathcal{B}}^{y}$ with $\mathcal{A}_{j}^{y}$.

Assume $\overline{\mathcal{B}}^{1}$ is of order $J$. In DCC, the secondary delivery set used for each round is a diagonal shift of the delivery set for the previous round. In fact, for every $y \in[2: P]$ we have

$$
\mathcal{A}_{j}^{y}=\left\{\left(\left(p_{i}+1\right) \% P,\left(k_{i}+\lambda\right) \% K\right) ; \forall\left(p_{i}, k_{i}\right) \in \mathcal{A}_{j}^{y-1}\right\},
$$

where $\%$ denotes the mod operator with offset one, defined as

$$
a \% b=((a-1) \bmod b)+1 .
$$

Based on this definition, $a \% a=a$ and $(a+b) \% a=b \% a$.

Example 5. Consider the network and the placement table in Example 1. As $\mathrm{T}$ is $a 4 \times 4$ table, we have $P=4$ and $\lambda=1$. Assume $\overline{\mathcal{B}}^{1}=\left\{\mathcal{A}_{1}^{1}, \mathcal{A}_{2}^{1}, \mathcal{A}_{3}^{1}\right\}$, where the primary delivery sets are equal to their counterparts in (11). Then we have $\overline{\mathcal{B}}^{2}=$ $\left\{\mathcal{A}_{1}^{2}, \mathcal{A}_{2}^{2}, \mathcal{A}_{3}^{2}\right\}$, where

$$
\begin{aligned}
& \mathcal{A}_{1}^{2}=\{(3,2),(2,3),(2,4)\}, \\
& \mathcal{A}_{2}^{2}=\{(4,2),(2,4),(2,1)\}, \\
& \mathcal{A}_{3}^{2}=\{(1,2),(2,1),(2,3)\},
\end{aligned}
$$

and the tabular view of $\overline{\mathcal{B}}^{2}$ is

$$
\mathrm{T}_{\overline{\mathcal{B}}^{2}}=\begin{array}{|l|l|l|l|}
\hline 0 & 1 & 0 & 0 \\
\hline 2 & 0 & 2 & 2 \\
\hline 0 & 1 & 0 & 0 \\
\hline 0 & 1 & 0 & 0 \\
\hline
\end{array},
$$

which is a diagonally shifted version of $\mathrm{T}_{\overline{\mathcal{B}}^{1}}$, as presented in (13). Following the same procedure, the tabular view of $\overline{\mathcal{B}}^{3}$ and $\overline{\mathcal{B}}^{4}$ would be

$$
\mathrm{T}_{\overline{\mathcal{B}}^{3}}=\begin{array}{|l|l|l|l}
\hline 0 & 0 & 1 & 0 \\
\hline 0 & 0 & 1 & 0 \\
\hline 2 & 2 & 0 & 2 \\
\hline 0 & 0 & 1 & 0 \\
\hline
\end{array}, \quad \mathrm{T}_{\overline{\mathcal{B}}^{4}}=\begin{array}{|l|l|l|l|l|}
\hline 0 & 0 & 0 & 1 \\
\hline 0 & 0 & 0 & 1 \\
\hline 0 & 0 & 0 & 1 \\
\hline 2 & 2 & 2 & 0 \\
\hline
\end{array} .
$$

The delivery procedure is completed by building a trans-

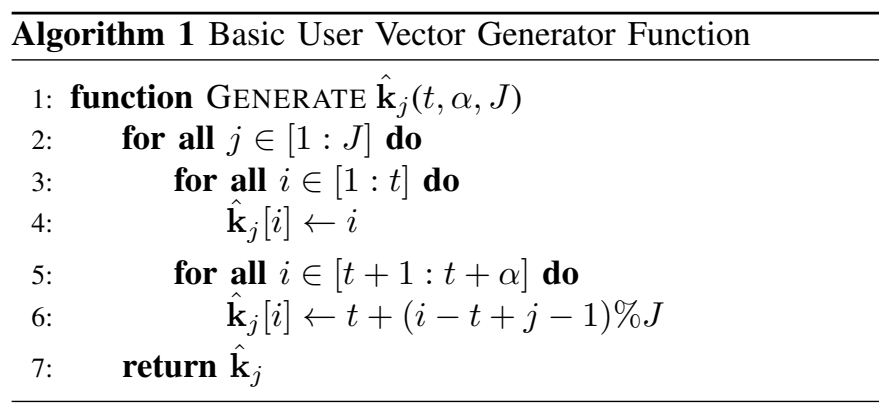

mission vector $\mathrm{x}\left(\mathcal{A}_{j}^{y}\right)$, for every $\mathcal{A}_{j}^{y} \in \overline{\mathcal{B}}^{y}$, for all $y \in[P]$. Overall, this indicates $J \times P$ transmissions, which are sent in consecutive time intervals. After each transmission is concluded, $t+\alpha$ users receive part of their requested data interference-free. The delivery result table, denoted by $\mathrm{T}_{R}$, is then built as

$$
\mathrm{T}_{R}=\mathrm{T}+\sum_{y=1}^{P} \mathrm{~T}_{\overline{\mathcal{B}} y} .
$$

Delivery scheme is called feasible, if all cells in $\mathrm{T}_{R}$, except the ones including $c$, have the same integer value of $Q$ (the second primal condition and the diagonal shifting structure guarantee that in $\mathrm{T}_{\mathcal{R}}$, each cell includes either $c$ or an integer number). In fact, according to (19), the value of $\mathrm{T}_{R}[(p, k)]$ indicates how many times $W_{p}(k)$ has appeared in the transmission vectors. So, if all the cells (except the ones including $c$ ) have the same value of $Q$, in the placement phase one can split every packet $W_{p}$, for every $W \in \mathcal{F}$ and $p \in[P]$, into $Q$ equal-sized subpackets $W_{p}^{q}$, and send a different subpacket every time $W_{p}$ appears in a transmission vector. The total subpacketization is then $S=P \times Q$.

Example 6. Consider the network ant the placement table in Example 1. Consider $\mathrm{T}_{\overline{\mathcal{B}} y}, y \in[4]$, to be as provided in Example 5. Then the delivery result table is

$$
\mathrm{T}_{R}=\begin{array}{|l|l|l|l|}
\hline c & 3 & 3 & 3 \\
\hline 3 & c & 3 & 3 \\
\hline 3 & 3 & c & 3 \\
\hline 3 & 3 & 3 & c \\
\hline
\end{array}
$$

which means the delivery scheme is feasible with $Q=3$. As $P=4$, this delivery scheme requires $S=12$.

\section{E. A Feasible Scheme}

Interestingly, the CC scheme presented in [11] is a feasible DCC scheme, applicable to any network with $\alpha \geq t$. In this scheme, $P=K$ (i.e. $\lambda=1$ ) and $Q=t+\alpha$ (i.e. $J=K-t$ ); and $\overline{\mathcal{B}}^{1}$ consists of primary delivery sets $\mathcal{A}_{j}^{1}, j \in[J]$, where

$$
\mathcal{A}_{j}^{1}=\left\{\left(\hat{\mathbf{p}}_{j}[i], \hat{\mathbf{k}}_{j}[i]\right) ; \forall i \in[t+\alpha]\right\} .
$$

In this equation, $\hat{\mathbf{k}}_{j}$ and $\hat{\mathbf{p}}_{j}$ are the basic user and packet index vectors built with Algorithms 1 and 2, respectively. It should be noted that the delivery procedure provided through Examples 1-6 in this paper is based on the CC scheme in [11].

\section{LOWER BOUND ON SUbPaCKETIZATION}

DCC requires subpacketization $S=P \times Q$, where $P$ can be any integer such that $\lambda=\frac{K}{P}$ and $\frac{t}{\lambda}$ are both integers. Clearly, 


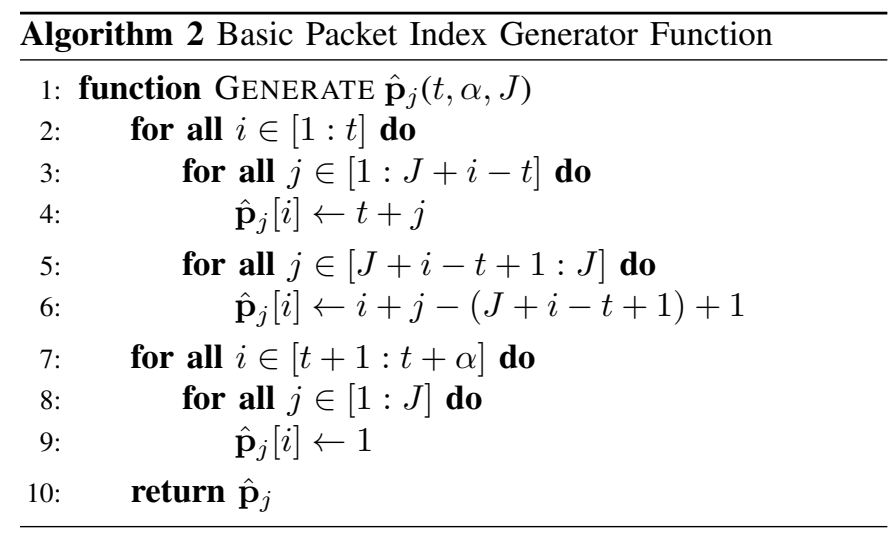

the minimum $P$ value satisfying both conditions is

$$
P_{\min }=\frac{K}{\operatorname{gcd}(K, t)},
$$

where $\operatorname{gcd}(K, t)$ is the greatest common divisor of $K$ and $t$.

Theorem 1. In DCC structure, regardless of $P$, the minimum possible value for $Q$ is given by

$$
Q_{\min }=\frac{t+\alpha}{\operatorname{gcd}(K-t, t+\alpha)} .
$$

Proof. If $\mathcal{A}_{j}^{y}$ is a primary delivery set, $\mathbf{x}\left(\mathcal{A}_{j}^{y}\right)$ delivers useful data chunks to $t+\alpha$ users. Hence, in $\mathrm{T}_{\mathcal{A}_{j}^{y}}$ there exist exactly $t+\alpha$ cells with value 1 . Moreover, a secondary delivery set $\overline{\mathcal{B}}^{y}$ consists of $J$ primary delivery sets $\mathcal{A}_{j}^{y}$, which means the sum of all cell values in $\mathrm{T}_{\overline{\mathcal{B}} y}$ is $J(t+\alpha)$.

On the other hand, the placement table $\mathrm{T}$ has $P \times K$ total cells, from which $P \times t$ cells are filled with $c$. So, T has $P(K-t)$ zero-valued cells. For the procedure to be feasible, in $\mathrm{T}_{R}$ all these cells should be filled with $Q$. However, there exist $P$ transmission rounds, and each of them increases the sum value in (19) by $J(t+\alpha)$. So we should have

$$
P(K-t) Q=P J(t+\alpha),
$$

and as a result

$$
Q=\frac{J(t+\alpha)}{K-t}
$$

As $Q$ needs to be an integer, the minimum value for $J$ is

$$
J_{\min }=\frac{K-t}{\operatorname{gcd}(K-t, t+\alpha)},
$$

which results in the $Q_{\min }$ value in (23)

Corollary 1. The subpacketization value for a DCC scheme cannot be smaller than

$$
S_{\min }=P_{\min } \times Q_{\min }=\frac{K}{g c d(K, t)} \frac{t+\alpha}{g c d(K-t, t+\alpha)} .
$$

Corollary 2. If $\operatorname{gcd}(K, t)=1$ and $\operatorname{gcd}(K-t, t+\alpha)=1$, the coded caching scheme in [11] is subpacketization-optimal among all DCC schemes.

\section{A. Achievability of the Lower Bound}

The $S_{\min }$ value in (27) is achievable for any network with $\alpha \geq t$. The secondary delivery set $\overline{\mathcal{B}}^{1}$ resulting in achievability consists of primary delivery sets $\mathcal{A}_{j}^{1}, j \in\left[J_{\text {min }}\right]$, built as

$$
\mathcal{A}_{j}^{1}=\left\{\left(\mathbf{p}_{j}[i], \mathbf{k}_{j}[i]\right) ; \forall i \in[t+\alpha]\right\},
$$
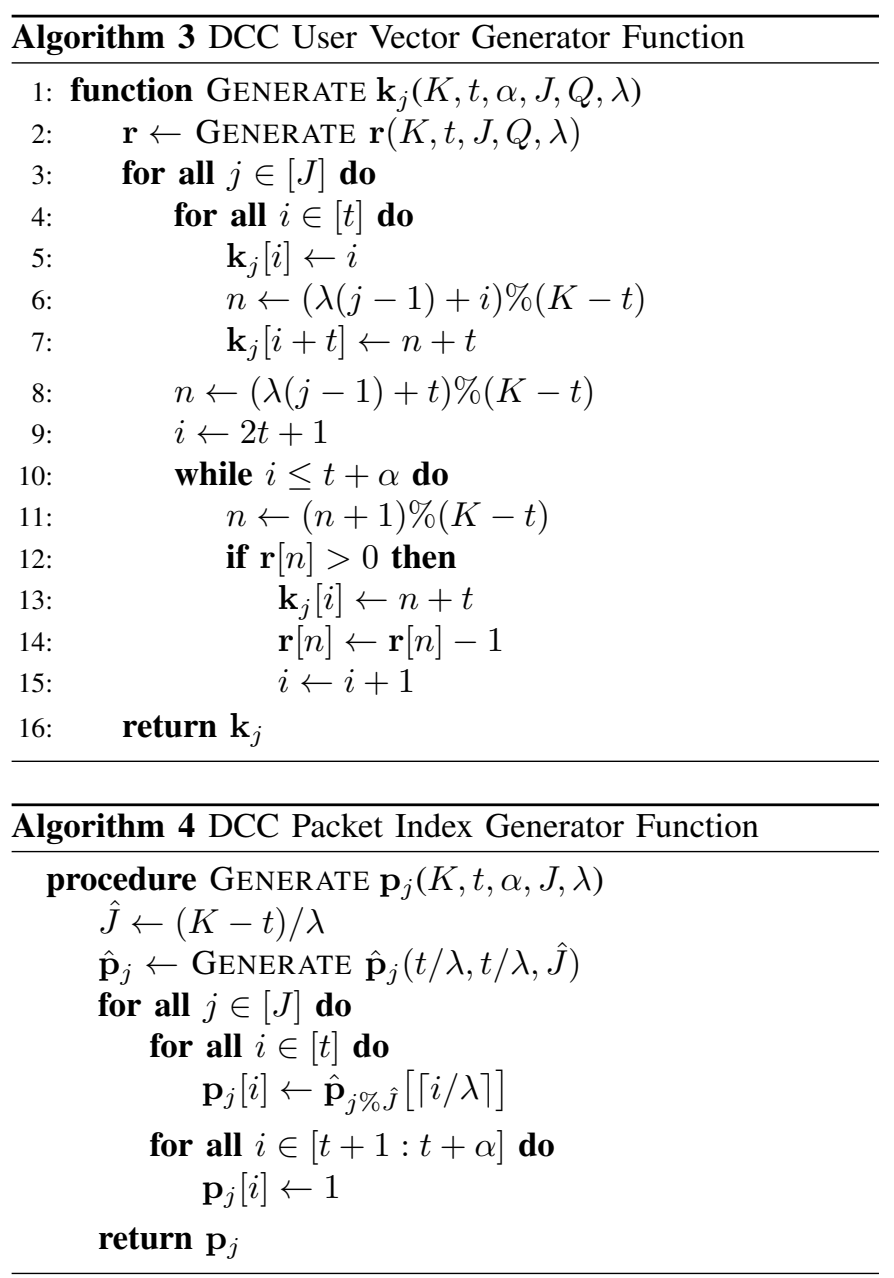

where $\mathbf{k}_{j}$ and $\mathbf{p}_{j}$ are the user and packet index vectors built using Algorithms 3 and 4, after setting $J=J_{\min }, Q=Q_{\min }$ and $\lambda=K / P_{\min }$ (note that these algorithms provide one achievable scheme, the solution may not be unique). As a brief explanation, the transmission vectors built by primary delivery sets $\mathcal{A}_{j}^{1}$ are no longer shifted versions of each other as in [11]. Instead, a new budget vector $\mathbf{r}$, built by Algorithm 5, guides the user vector generation process in Algorithm 3. In fact, $\mathbf{r}$ indicates how many times each user should appear in one of the transmission vectors in the first transmission round, for the scheme to become feasible. The elements in $\mathbf{r}$ are updated after each iteration in Algorithm 3, until all the transmission vectors of the first round are built. Due to the lack of space, a more thorough explanation of the intuition behind Algorithms 3-5 is left for the extended version of this paper $^{3}$.

Example 7. Consider a network with $K=6, t=2$ and $\alpha=2$. For this network, the subpacketization requirement for the multi-server scheme in [7] is $\left(\begin{array}{l}6 \\ 2\end{array}\right)\left(\begin{array}{l}3 \\ 1\end{array}\right)=45$, while in [11] it is reduced to $6 \times 4=24$. However, using the new scheme in this paper, we have $P_{\min }=3$ and $Q_{\min }=1$, resulting in $S_{\min }=3$. In order to achieve this, as $\lambda=K / P_{\min }=2$, the

\footnotetext{
${ }^{3}$ For interested readers, MATLAB codes of the algorithms are publicly available in: https://bit.ly/2TOZnfn
} 


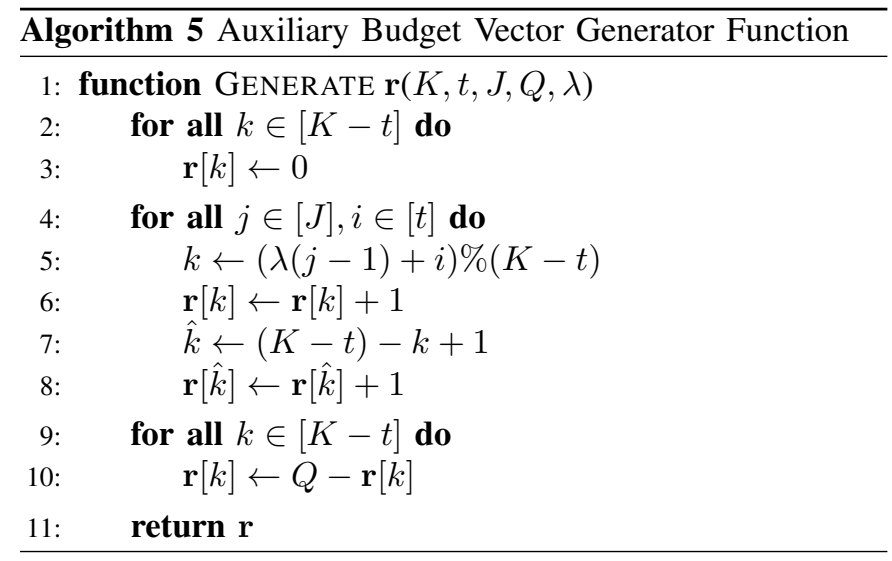

placement table is built as

$$
\mathrm{T}=\begin{array}{|l|l|l|l|l|l|}
\hline c & c & 0 & 0 & 0 & 0 \\
\hline 0 & 0 & c & c & 0 & 0 \\
\hline 0 & 0 & 0 & 0 & c & c \\
\hline
\end{array}
$$

and $\hat{\mathcal{B}}^{1}$ consists of only one primary delivery set $\mathcal{A}_{1}^{1}$, built as

$$
\mathcal{A}_{1}^{1}=\{(2,1),(2,2),(1,3),(1,4)\} .
$$

Data delivery is performed with three transmissions. Assuming the demand set is $\mathcal{D}=\{A, B, C, D, E, F\}$, we have

$$
\begin{aligned}
& \mathbf{x}\left(\mathcal{A}_{1}^{1}\right)=\mathbf{w}_{2} A_{2}^{1}+\mathbf{w}_{1} B_{2}^{1}+\mathbf{w}_{4} C_{1}^{1}+\mathbf{w}_{3} D_{1}^{1}, \\
& \mathbf{x}\left(\mathcal{A}_{1}^{2}\right)=\mathbf{w}_{4} C_{3}^{1}+\mathbf{w}_{3} D_{3}^{1}+\mathbf{w}_{6} E_{2}^{1}+\mathbf{w}_{5} F_{2}^{1}, \\
& \mathbf{x}\left(\mathcal{A}_{1}^{3}\right)=\mathbf{w}_{6} E_{1}^{1}+\mathbf{w}_{5} F_{1}^{1}+\mathbf{w}_{2} A_{3}^{1}+\mathbf{w}_{1} B_{3}^{1} .
\end{aligned}
$$

It is noteworthy that although $K$ and $t$ are predefined by the user count, cache size, and the file library size parameters, $\alpha$ is a design parameter which can be any integer in $[t: L]$. In addition to the current practice of selecting $\alpha$ to balance between the spatial DoF and the beamforming gain as outlined in [12], one can also tune it to achieve smaller subpacketization for a given network. In Table I we have compared the required subpacketization for different schemes, for various $K, t, \alpha$ values. In this table, DCC, LIN, and MS refer to the new scheme presented in this paper, the linear scheme in [11] and the original multi-server scheme in [7], respectively. As can be seen, except for a few specific cases in very small networks (e.g., the network in the first row of the table), the new scheme results in considerable reductions in the required subpacketization.

\begin{tabular}{|c|c|c||c|c|c|}
\hline$K$ & $t$ & $\alpha$ & DCC & LIN & MS \\
\hline \hline 4 & 1 & 1 & 8 & 8 & 4 \\
\hline 4 & 1 & 2 & 4 & 12 & 8 \\
\hline 8 & 2 & 3 & 20 & 40 & 180 \\
\hline 8 & 2 & 4 & 4 & 48 & 280 \\
\hline 8 & 2 & 5 & 28 & 56 & 140 \\
\hline 12 & 3 & 4 & 28 & 84 & $>10^{4}$ \\
\hline 12 & 3 & 5 & 32 & 96 & $>10^{4}$ \\
\hline 12 & 5 & 6 & 132 & 132 & 4752 \\
\hline 20 & 5 & 5 & 8 & 200 & $>10^{7}$ \\
\hline 20 & 5 & 7 & 16 & 240 & $>10^{7}$ \\
\hline 20 & 5 & 10 & 4 & 300 & $>10^{7}$ \\
\hline 30 & 5 & 5 & 12 & 300 & $>10^{9}$ \\
\hline 30 & 5 & 10 & 18 & 450 & $>10^{11}$ \\
\hline 30 & 5 & 15 & 24 & 600 & $>10^{11}$ \\
\hline
\end{tabular}

TABLE I: Required Subpacketization for Different Schemes

\section{CONCLUSIONS AND Future WORK}

In this paper, we proposed a new class of diagonal coded caching schemes, based on the diagonal shifting of transmitted codewords over a tabular structure. We also found the lowerbound for the required subpacketization in this class and provided new algorithms to achieve that. This not only enables smaller subpacketization compared to state of the art but also makes it possible to control the subpacketization by adjusting $\alpha$. Analyzing the provided schemes' performance at finiteSNR and extending their applicability to the $t>\alpha$ regime are parts of the ongoing research.

\section{REFERENCES}

[1] V. N. I. Cisco, "Cisco visual networking index: Forecast and trends, 2017-2022," White Paper, vol. 1, 2018.

[2] V. Jacobson, D. K. Smetters, J. D. Thornton, M. Plass, N. Briggs, and R. Braynard, "Networking named content," in Communications of the $A C M$, vol. 55, no. 1, 2012, pp. 117-124.

[3] M. Cha, H. Kwak, P. Rodriguez, Y. Y. Ahn, and S. Moon, "Analyzing the video popularity characteristics of large-scale user generated content systems," IEEE/ACM Transactions on Networking, vol. 17, no. 5, pp. 1357-1370, 2009.

[4] M. J. Salehi, S. A. Motahari, and B. H. Khalaj, "On the optimality of 0-1 data placement in cache networks," IEEE Transactions on Communications, vol. 66, no. 3, pp. 1053-1063, 2018.

[5] A. Leventhal, "Flash storage memory," Communications of the ACM, vol. 51, no. 7, pp. 47-51, 2008.

[6] M. A. Maddah-Ali and U. Niesen, "Fundamental limits of caching," IEEE Transactions on Information Theory, vol. 60, no. 5, pp. 28562867, 2014.

[7] S. P. Shariatpanahi, S. A. Motahari, and B. H. Khalaj, "Multi-server coded caching," IEEE Transactions on Information Theory, vol. 62, no. 12, pp. 7253-7271, 2016.

[8] S. P. Shariatpanahi, G. Caire, and B. Hossein Khalaj, "PhysicalLayer Schemes for Wireless Coded Caching," IEEE Transactions on Information Theory, vol. 65, no. 5, pp. 2792-2807, 2019.

[9] N. Rajatheva, I. Atzeni, E. Bjornson, A. Bourdoux, S. Buzzi, J.-B. Dore, S. Erkucuk, M. Fuentes, K. Guan, Y. Hu, and others, "White Paper on Broadband Connectivity in 6G," arXiv preprint arXiv:2004.14247, 2020.

[10] E. Lampiris and P. Elia, "Adding transmitters dramatically boosts codedcaching gains for finite file sizes," IEEE Journal on Selected Areas in Communications, vol. 36, no. 6, pp. 1176-1188, 2018.

[11] M. Salehi, A. Tölli, and S. P. Shariatpanahi, "A Multi-Antenna Coded Caching Scheme with Linear Subpacketization," in 2020 IEEE International Conference on Communications (ICC), 2019, pp. 1-6.

[12] A. Tolli, S. P. Shariatpanahi, J. Kaleva, and B. H. Khalaj, "Multi-antenna interference management for coded caching," IEEE Transactions on Wireless Communications, vol. 19, no. 3, pp. 2091-2106, 2020.

[13] M. J. Salehi, A. Tolli, and S. P. Shariatpanahi, "Subpacketizationbeamformer interaction in multi-antenna coded caching," in 2nd $6 G$ Wireless Summit 2020: Gain Edge for the 6G Era, 6G SUMMIT 2020, 2020, pp. 1-5.

[14] Q. Yan, M. Cheng, X. Tang, and Q. Chen, "On the placement delivery array design for centralized coded caching scheme," IEEE Transactions on Information Theory, vol. 63, no. 9, pp. 5821-5833, 2017.

[15] Q. Yan, X. Tang, Q. Chen, and M. Cheng, "Placement Delivery Array Design Through Strong Edge Coloring of Bipartite Graphs," IEEE Communications Letters, vol. 22, no. 2, pp. 236-239, 2018.

[16] C. Shangguan, Y. Zhang, and G. Ge, "Centralized coded caching schemes: A hypergraph theoretical approach," IEEE Transactions on Information Theory, vol. 64, no. 8, pp. 5755-5766, 2018.

[17] K. Shanmugam, A. M. Tulino, and A. G. Dimakis, "Coded caching with linear subpacketization is possible using Ruzsa-Szeméredi graphs," in IEEE International Symposium on Information Theory - Proceedings. IEEE, 2017, pp. 1237-1241.

[18] M. Salehi, A. Tolli, S. P. Shariatpanahi, and J. Kaleva, "Subpacketization-rate trade-off in multi-antenna coded caching," in 2019 IEEE Global Communications Conference, GLOBECOM 2019 - Proceedings. IEEE, 2019, pp. 1-6. 